\title{
New Algorithm for Detecting Target on the Background Clutter Using Polarimetric Parameters
}

\author{
Hung Trong Pham, Nghia Minh Pham, Thanh Trung Nguyen \\ Faculty of Radio-Electronics, Le Quy Don University, Hanoi, Vietnam \\ Correspondence: Thanh Trung Nguyen, thanhmta@gmail.com \\ Communication: received 21 April 2016, revised 29 June 2016, accepted 30 December 2016 \\ Online publication: 9 February 2017, Digital Object Identifier: 10.21553/rev-jec.112 \\ The associate editor coordinating the review of this article and recommending it for publication was Prof. Nguyen Linh-Trung.
}

\begin{abstract}
This paper proposes a new algorithm for detecting radar targets on the background clutter using a non-energy polarimetry parameter, the elliptic coefficient. The probability density function of the elliptic coefficient is calculated for two cases: target in clutter and only clutter. The algorithm ultilises a detector with two-level threshold which is calculated based on the Neyman-Pearson criteria. In this paper, the detection threshold and the probability of detection are calculated based on a given probability of false alarm, different signal to background clutter ratios, and with different polarimetric features of the background clutter. Simulation results of the proposed algorithm shows the potential application of using polarimetric elliptic coefficient in detecting small radar targets on the background clutter.
\end{abstract}

Keywords- Polarimetric radar, small target detection, elliptic coefficient, two-level threshold.

\section{INTRODUCTION}

The detection of the target based on threshold is commonly used in radar. In that, the detection characteristics, the probability of false alarm $\left(P_{F}\right)$ and the probability of detection $\left(P_{D}\right)$, are determined from the signal to noise ratio (SNR) and the optimum threshold selection criteria.

In polarimetric radars, the detection is more complicated because there are 2 signals at the output of the antenna system, both of them need to be examined for an accurate detection. The utilization of parameters extracted from the scattering matrix (SM) or invariant parameters of the SM will increase $P_{D}$ compared to the case where parameters are only determined based on echoes from the radar cross section of target using a single channel. The polarimetric information can be utilized in radar applications such as: detection, classification, identification, target selection, to name a few.

The detection of small scale targets in background clutter (sea surface, ground, etc.) is often complicated, especially when the echoes from the targets are weak as compared to the background clutter. In such cases, the utilization of non-energy polarimetric parameters has shown promising results. In recent years, several algorithms of detecting small targets on the background clutter using polarimetric information have been proposed. This brings a new prospect in using polarimetric radars for detecting small targets. Vachula [1] developed statistical models which calculate all changes in the polarization of an incident radar signal upon reflection. He used these models to analyze a detector in which thresholds are various functions of the polarization-related parameters known as Stock parameters [2]. In [3] and [4], Gromov used the elliptic angle coefficient for a non-energy detection and selection of signals from ground-based radars by passive radars on satellites. Those papers showed potential applications of polarimetric parameters in the radar detection, and especially, in the target selection. In [5], Karnjsev used the complex degree of anisotropy for detection, and this parameter is calculated based on the linear polarization basic where the circular polarization basic is perhaps the optimum solution and should be used [6,7]. The author of [5] proposed the use of polarimetric ratio for the detection, in that the priori information of the probability density function must be known. Papers [8] and [9], from experiment results, proved that the 'polarization trace' does exist. The 2 papers also proposed the use of this parameter for the detection of small scale targets in the background clutter, especially in the sea clutter. In the newest research on detection of target in the background clutter based on the polarimetric parameter [7], this parameter had not been used for a particular application, but only for representing the sign of target on the radar image.

This paper proposes a novel algorithm in which the elliptic coefficient is used for the detection based on the Neyman-Pearson criteria. In this paper, the detection threshold and $P_{D}$ are calculated based on a given $P_{F}$. A two-level threshold is applied for the detection of small target in background clutter based on polarimetric parameters.

The remainder of this paper is organized as follows: Section 2 discusses the elliptic coefficient, Section 3 presents the statistical characteristics of the elliptic coefficient based on circular polarization basic. Section 4 investigates the use of the elliptic coefficient for the target detection. The conclusion is given in Section 5 . 


\section{The Elliptic Coefficient}

In polarimetric radars, the main idea of scattered signals polarization parameters utilization is the use of the radar objects polarization scattering matrix (PSM) invariants. These invariants are the combinations of PSM eigenvalues, which can be measured directly by the simple algorithm in [6]. Measurements of two invariants are sufficient: a full power of scattered signal and a module of complex degree polarization anisotropy (CDPA) of a radar object. The last parameter is closely connected to the elliptic polarization coefficient of the scattered wave [6]. The measurements of the invariants for every cell of the radar map can be implemented in real time.

It was demonstrated in [6] that when a right-hand circular polarization (RHCP) signal is radiated, a circular polarization ratio of scattered left-hand circular polarization (LHCP) and RHCP signals equals the CDPA of radar object:

$$
\begin{aligned}
p_{\dot{R} L} & =\frac{\cos \alpha+\sin \alpha}{\cos \alpha-\sin \alpha} \times e^{-i \times\left(2 \beta-\frac{\pi}{2}\right)} \\
& =\tan \left(\alpha+\frac{\pi}{4}\right) \times e^{-i \times\left(2 \beta-\frac{\pi}{2}\right)},
\end{aligned}
$$

where $\alpha, \beta$ are the elliptic angle and the orientation angle of the polarization ellipse. A CDPA module is closely connected to radar object polarization properties, as in [6]:

$$
\left|p_{\dot{R} L}\right|=|\dot{\mu}|=\left|\frac{\dot{\lambda_{1}}-\dot{\lambda_{2}}}{\dot{\lambda_{1}}+\dot{\lambda_{2}}}\right|=\tan \left(\alpha+\frac{\pi}{4}\right),
$$

where $\dot{\lambda_{1}}, \dot{\lambda_{2}}$ are the eigenvalues of the radar object PSM. However, the use of CDPA module is complicated, because this value is determined on the interval $0<|\dot{\mu}|<\infty$. We will carry out the linear-fractional transformation of the CDPA module as follow:

$$
\frac{\left|p_{\dot{R} L}\right|-1}{\left|\dot{p}_{R L}\right|+1}=\frac{\tan \left(\alpha+\frac{\pi}{4}\right)-1}{\tan \left(\alpha+\frac{\pi}{4}\right)+1}=\tan \alpha=K,
$$

where $K,\left|p_{\dot{R} L}\right|=\left|\frac{E_{L}}{E_{R}}\right|$ are the elliptic coefficient and the circular polarization ratio of the scattered wave respectively. It can be seen that $\mathrm{K}$ is determined by the eigenvalues of the radar object PSM. Therefore, the radar object polarization properties are reflected in the measured value directly. It is necessary to point out that, at present, radar systems do not measure radar object polarization properties directly. Instead, the elliptic angle is determined on the interval $-\pi / 4<\alpha<\pi / 4$, and then the elliptic coefficient is determined on the interval $-1 \leq K \leq 1$ accordingly. In this case, $K$ receivers values $-1,0$, and 1 when the radar object are a trihedral corner reflector, a dipole, and a dihedral corner reflector, respectively [6].

In the next section, we will present the statistical characteristics of the elliptic coefficient based on circular polarization basic and the capability of using this polarimetric parameter to detect small targets on the background clutter.

\section{Statistical Characteristics of Elliptic Coefficient based on the Circular Polarization Basic}

For the target detection based on polarimetric parameters, the statistical models of signals and background clutter are necessary. Those models are well presented in [10].

In the radar applications $[8,9,11]$, the transmitted signal is RHCP, the received signal is simultaneously combined from RHCP and LHCP channels. The system then determines the absolute value of the polarization ratio as follow:

$$
\left|\dot{p}_{R L}(t)\right|=\left|\frac{\dot{E_{L}}(t)}{\dot{E_{R}}(t)}\right|
$$

The elliptic coefficient is calculated as in [8]:

$$
K(t)=\frac{\left|p_{\dot{R} L}(t)\right|-1}{\left|p_{\dot{R} L}(t)\right|+1},-1 \leq K \leq 1
$$

Simulation results with the target echoes from the range gate 2 to $4 \mu$ s are illustrated in Figures 1 and 2 . The results comes with assumptions that $E_{R}(t)$ and $E_{L}(t)$ components of the background clutter are statistical independence and they both have Gaussian distribution with zero means. Figure 1 illustrates the received signals from RHCP and LHCP channels. Figure 2 presents the evaluated values of $K$. Mean value $m_{K}$ of $K$ for the background clutter approximately 0 , and for the target in the background clutter nearly $-0,4$.

From [10], the general formula for the probability density function (PDF) of the elliptic coefficient, $W(K)$, in the circular polarization basic with the RHCP transmitted signal is complicated. In this paper, for simplicity, the authors calculate for a special case, where there is no correlation between orthogonal polarization components, $R=0$. The PDF of $W(K)$ in this case is given in (6), where $a_{i}^{2}=\frac{E_{0 i}^{2}}{\sigma_{i}^{2}}$ - target to clutter power ratio (TCR), in that $i=2$ for the LHCP channel, and $i=1$ for the RHCP channel; $b=\frac{a_{2}}{a_{1}}=\frac{E_{0 R}}{E_{0 L}} \frac{\sigma_{L}}{\sigma_{R}}=$ $\left|P_{0 R L}\right| \frac{\sigma_{L}}{\sigma_{R}}$ - the parameter characterizes the total signal, scattering both from target + background clutter, and $P_{O R L}$ characterizes the polarimetric feature of the target; $h^{2}=\frac{\sigma_{L}^{2}}{\sigma_{R}^{2}}$ - characterizes the polarimetric feature of the background clutter.

The PDF of the polarimetric parameter $K$ on the circular polarization basic, therefore, depends on the following variables: $a_{1}, a_{2}$ - characterizes signals on the 2 orthogonal polarization channels; $b$ - characterizes the polarimetric feature of the total signal; and $h, \sigma_{L}$, $\sigma_{R}$ - characterizes the feature of the background clutter. The dependence of the PDF of $W(K)$ in Equation (6) on variables $a_{1}, b, h$ are illustrated in Figures 3,4 , and 5 .

As can be seen from Figure 3, with $R=0, a_{1}=0.3$, $b=5$, different values of $h$ represent different types of background clutter. As $h$ increases from 1 to $\infty, m_{K}$ approaches -1 . As $h$ decreases from 1 to $0, m_{K}$ approaches +1 . When $h=1$, the PDF $W(K)$ is a symmetric function in the domain $K \in[-1,1]$. In the target detection, only 


$$
\begin{aligned}
W\left(K, a_{1}, b, h\right)= & \frac{4 h^{2}\left(1-K^{2}\right)}{\left[(1-K)^{2}+h^{2}(1+K)^{2}\right]^{2}} \times \exp \left\{-\frac{a_{1}^{2}\left[(1-K)^{2} b^{2}+(1+K)^{2} h^{2}\right]}{2\left[(1-K)^{2}+h^{2}(1+K)^{2}\right]}\right\} \\
& \times\left\{\left[1+\frac{a_{1}^{2}\left[(1-K)^{2}+(1+K)^{2} h^{2} b^{2}\right]}{2\left[(1-K)^{2}+(1+K)^{2} h^{2}\right]}\right] \times I_{0}\left[\frac{a_{1}^{2} b h(1-K)^{2}}{(1-K)^{2}+(1+K)^{2} h^{2}}\right]\right. \\
& \left.+\frac{a_{1}^{2} b h(1-K)^{2}}{(1-K)^{2}+(1+K)^{2} h^{2}} \times I_{1}\left[\frac{a_{1}^{2} b h(1-K)^{2}}{(1-K)^{2}+(1+K)^{2} h^{2}}\right]\right\}
\end{aligned}
$$
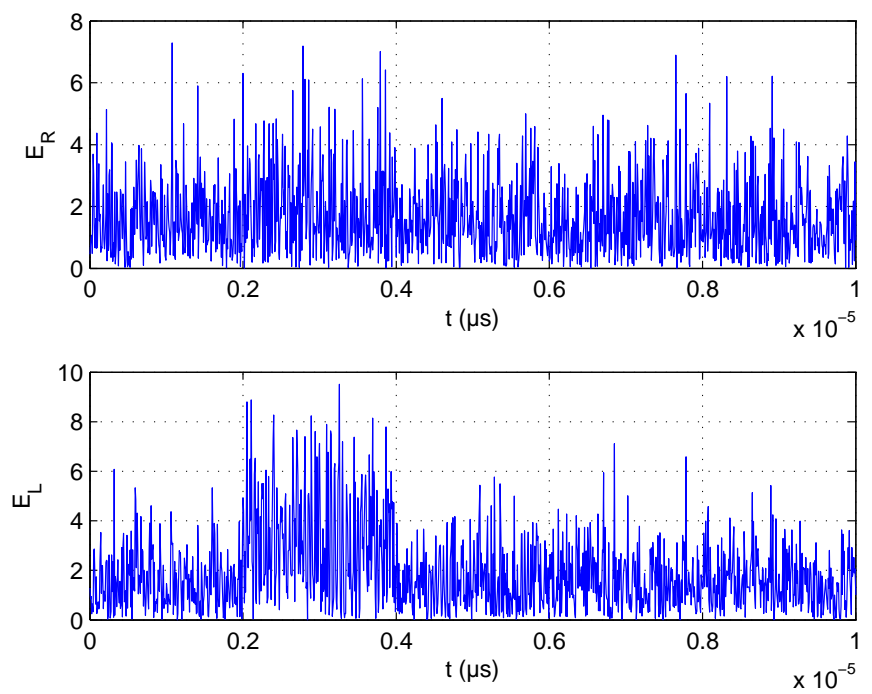

Figure 1. Received signals from right hand and left hand circular polarization channels

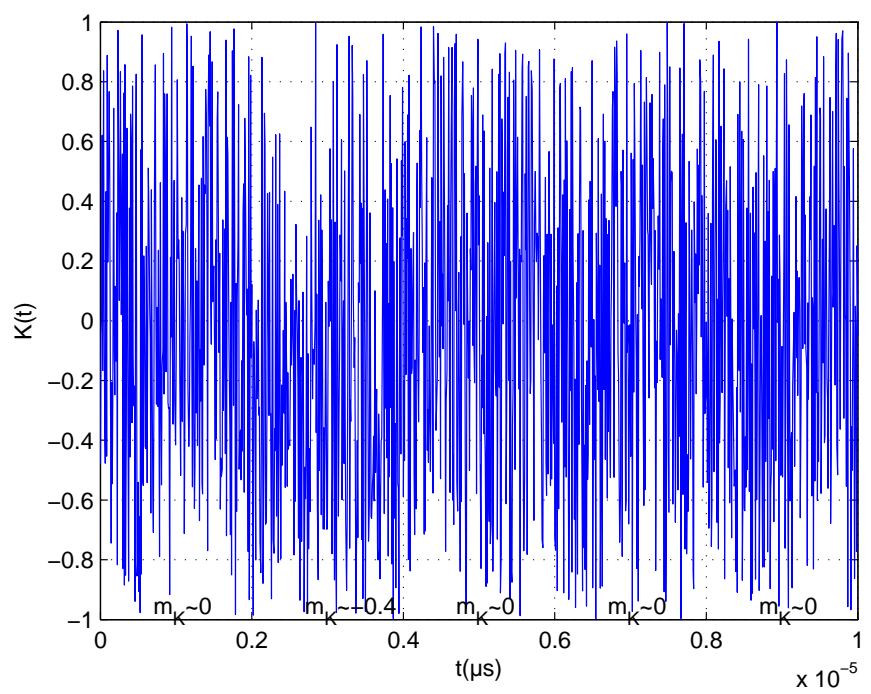

Figure 2. Evaluated values of the elliptic coefficient $K$

one value of $h$ is needed, corresponding to one type of background clutter.

Figure 4 shows that, with $R=0, h=1, a_{1}=1$, the PDF of $K$ changes significantly as the polarimetric characteristic of target, $b$ varies. As $b$ increases, $m_{K}$ approaches 1 . This happens when the radar object in the form of dihedral corner reflector. In contrast, as $b$ decreases to zero, $m_{K}$ approaches -1 , which happens when the radar object is a trihedral corner reflector.

With $R=0, h=1, b=5$, Figure 5 demonstrates the

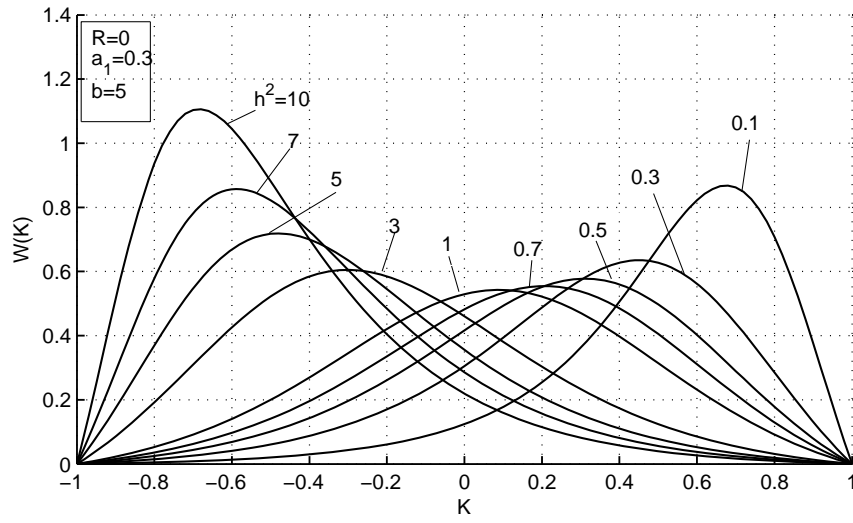

Figure 3. The dependence of the PDF $\mathrm{W}(\mathrm{K})$ on $h^{2}$ with $R=0, a_{1}=$ $0.3, b=5$.

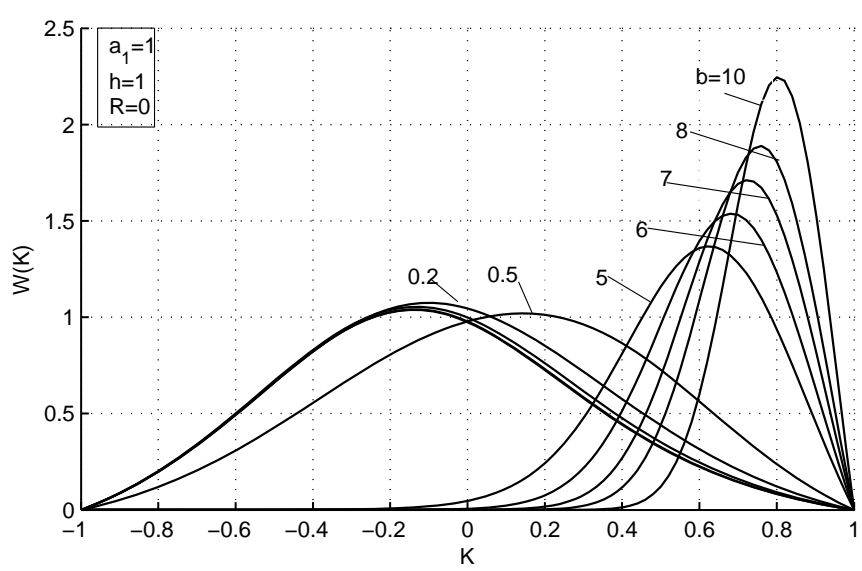

Figure 4. The dependence of the PDF $\mathrm{W}(\mathrm{K})$ on $\mathrm{b}$ with $R=0, h=$ $1, a_{1}=1$.

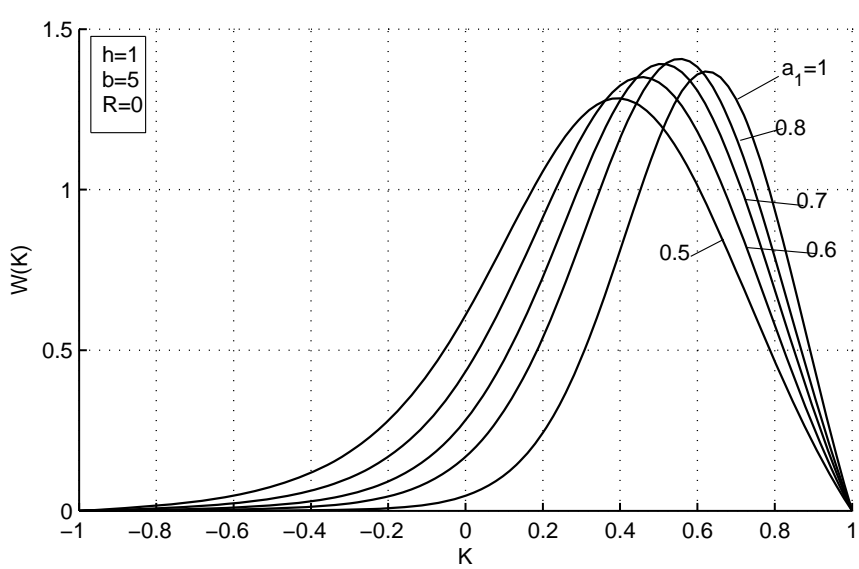

Figure 5. The dependence of the PDF $\mathrm{W}(\mathrm{K})$ on $a_{1}$ with $\mathrm{R}=0, \mathrm{~h}=$ $1, b=5$. 


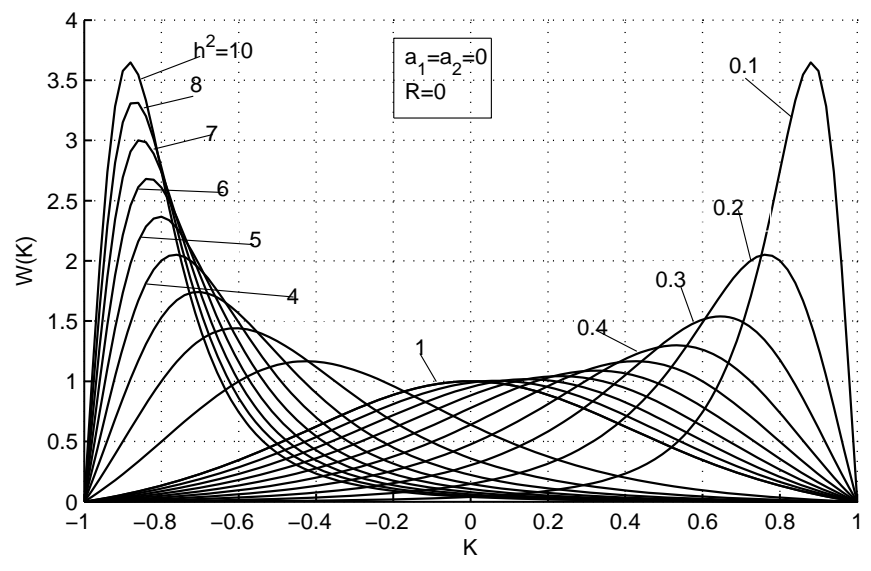

Figure 6. The PDF of the elliptic coefficient $\mathrm{K}$ with different $h^{2}$ when $R=0, a_{1}=a_{2}=0$

dependence of the PDF $W(K)$ on $a_{1}$, which is the TCR in the RHCP channel. As $a_{1}$ increases the PDF $W(K)$ becomes narrower, and $m_{K}$ approaches the polarimetric parameter of target. As $a_{1}$ decreases, the PDF $W(K)$ becomes wider, and $m_{K}$ approaches the polarimetric parameter of the background clutter.

If there is no target in the background clutter, $a_{1}=$ $a_{2}=0$, the orthogonal polarization components of the background clutter follow the Rayleigh distribution, Equation (6) will becomes:

$$
\begin{aligned}
& W(K, R, h) \\
& =\frac{4\left(1-R^{2}\right) h^{2}\left(1-K^{2}\right)\left[(1-K)^{2}+(1+K)^{2} h^{2}\right]}{\left\{\left[(1-K)^{2}+(1+K)^{2} h^{2}\right]-4 R^{2} h^{2}\left(1-K^{2}\right)^{2}\right\}^{3 / 2}}
\end{aligned}
$$

From Equation (7), the dependence of the PDF of $K$ on variables $R, h$ is illustrated in Figure 6 .

Figure 6 shows that, when there is no target on the background $a_{1}=a_{2}=0$ and $R=0$, as $h$ increased, the PDF $W(K)$ moves to the left side, and vice versa. When $h=1, W(K)$ is a standard distribution.

For the sea clutter, paper [12] shows that $\sigma_{L}^{2} \approx \sigma_{R}^{2}$. This is also confirmed by the experiment results in [7, 9]. Therefore, $h^{2}=\frac{\sigma_{L}^{2}}{\sigma_{R}^{2}} \approx 1$ and $R=0$ in this case. As a results, the PDF of $K$ of the sea clutter is a symmetric function in the domain $K \in(-1,1)$. The Equation (7) now becomes:

$$
W(K, R=0, h=1)=\frac{1-K^{2}}{\left(1+K^{2}\right)^{2}}
$$

The PDF of $K$ of the sea clutter is shown in Figure 7.

\section{The Target Detection based on Elliptic COEFFiCIENT}

From Equations (6) and (8), the PDF $W(K)$ of $K$ can be built on both 2 cases: only sea clutter exists - $W_{s c}(K)$ and there is a target in sea clutter $-W_{s c+t}(K)$. They are shown in Figure 8.

The detection algorithm is as follow: within the domain of $K$ from $[-1,+1]$, an interval of $K$ for the sea clutter $\Delta K_{s c}=\left[K_{L}, K_{R}\right]$ is set. Let we assume the value of the measured $K$, which corresponds to one radar cell,

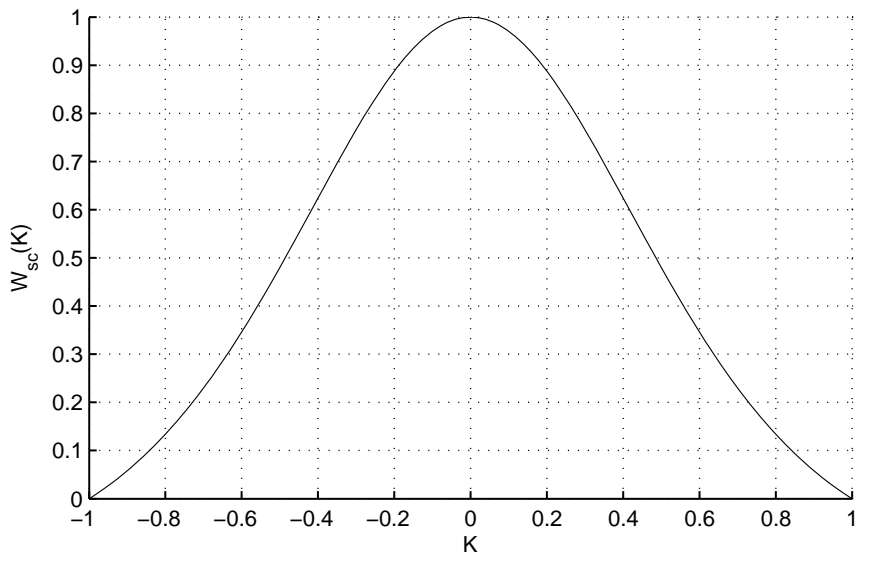

Figure 7. The PDF of $\mathrm{K}$ of the sea clutter with $R=0, h^{2}=1$

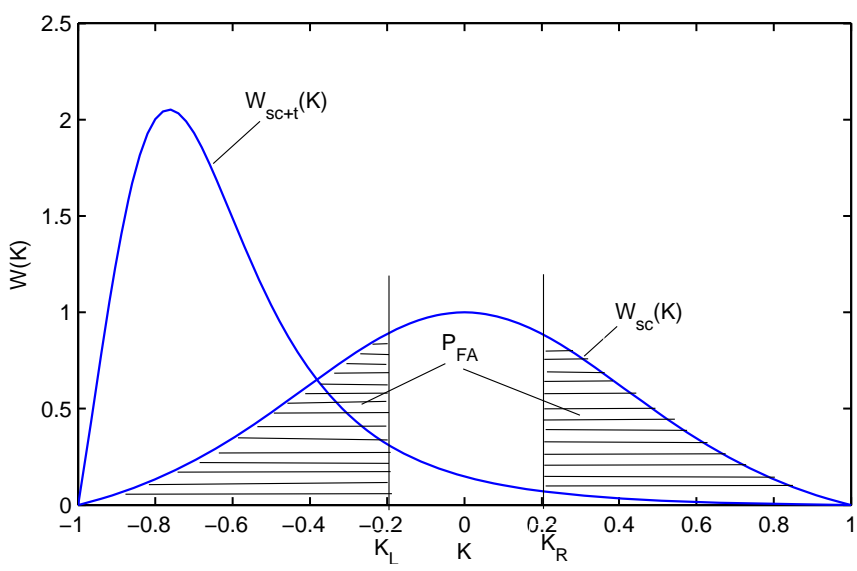

Figure 8. The difference between PDFs of $\mathrm{K}$ in two cases: 1. target in sea clutter, and 2 . only sea clutter

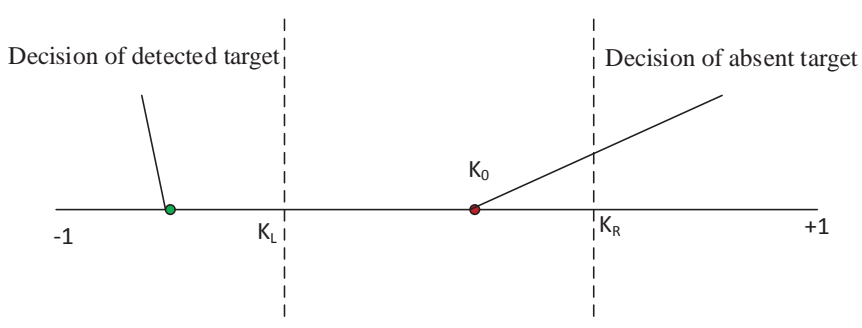

Figure 9. The decision of detection based on measured elliptic coefficient, $K$

is $K_{0}$. If $K_{0}$ is out of the interval $\left[K_{L}, K_{R}\right]$, or in other words $K_{0} \in\left(\left(-1, K_{L}\right) \cup\left(K_{R}, 1\right)\right)$, we then decide that there is a target within that radar cell. Otherwise, the decision is no target. The decision of detection based on measured elliptic coefficient is illustrated in Figure 9.

If the Neyman-Pearson criteria is used for determining the detection interval (the two-level threshold), the interval $\Delta K_{s c}$ is chosen so that the probability of the event $K \in\left(\left(-1, K_{L}\right) \cup\left(K_{R}, 1\right)\right)$ is equal $P_{F}$. The area within the detection interval and the function $W_{s c}(K)$ corresponds to the given $P_{F}$ and equals the marked area in Figure 8.

$$
P_{F}=P_{F 1}+P_{F 2}=\int_{-1}^{K_{L}} \mathrm{~W}_{s c}(K) \mathrm{d} K+\int_{K_{R}}^{1} \mathrm{~W}_{s c}(K) \mathrm{d} K
$$




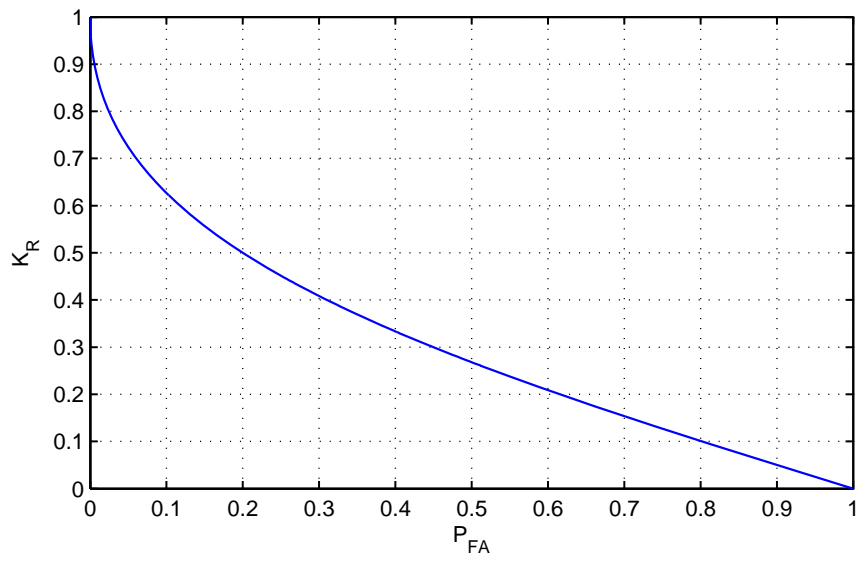

Figure 10. The dependence of the threshold $K_{R}$ on a given probability of false alarm

Table I

The Threshold $K_{R}$ Corresponding to Several Given $P_{F}$

\begin{tabular}{|l|l|}
\hline$P_{F}$ & Detection threshold range \\
\hline 0.1 & {$[-0.6,0.6]$} \\
0.2 & {$[-0.5,0.5]$} \\
0.3 & {$[-0.4,0.4]$} \\
0.4 & {$[-0.34,0.34]$} \\
0.5 & {$[-0.27,0.27]$} \\
\hline
\end{tabular}

With an assumption that the PDF of the sea clutter is as described in Figure 5, $K_{L}$ and $K_{R}$ can be considered additive inverses, or $K_{L}=-K_{R}$. Therefore, Equation (9) can be rewritten as follow:

$$
P_{F}=P_{F 1}+P_{F 2}=2 \int_{K_{R}}^{1} \mathrm{~W}_{s c}(K) \mathrm{d} K
$$

The probability of missed detection is

$$
P_{M}=\int_{K_{L}}^{K_{R}} \mathrm{~W}_{s c+t}(K) \mathrm{d} K .
$$

The probability of detection is

$$
P_{D}=1-P_{M}=1-\int_{K_{L}}^{K_{R}} \mathrm{~W}_{s c+t}(K) \mathrm{d} K,
$$

where $W_{s c+t}(K)$ is calculated from the equation (6). With the PDF of $K$ of the sea clutter as described in equation (8) and a given $P_{F}$, the detection threshold can be determined by solving the following equation:

$$
\begin{gathered}
P_{F}=2 \int_{K_{R}}^{1} \mathrm{~W}_{s c}(K) \mathrm{d} K=2 \int_{K_{R}}^{1} \frac{1-\mathrm{K}^{2}}{\left(1+\mathrm{K}^{2}\right)^{2}} \mathrm{~d} K \\
P_{F}=1-\left|\sin \left(2 \arctan K_{R}\right)\right|
\end{gathered}
$$

From Equation (14), the threshold $K_{R}$ is

$$
K_{R}=\tan \left[\frac{\arcsin \left(1-P_{F}\right)}{2}\right]
$$

The dependence of the threshold $K_{R}$ on $P_{F}$ is illustrated in Figure 10 and Table I.

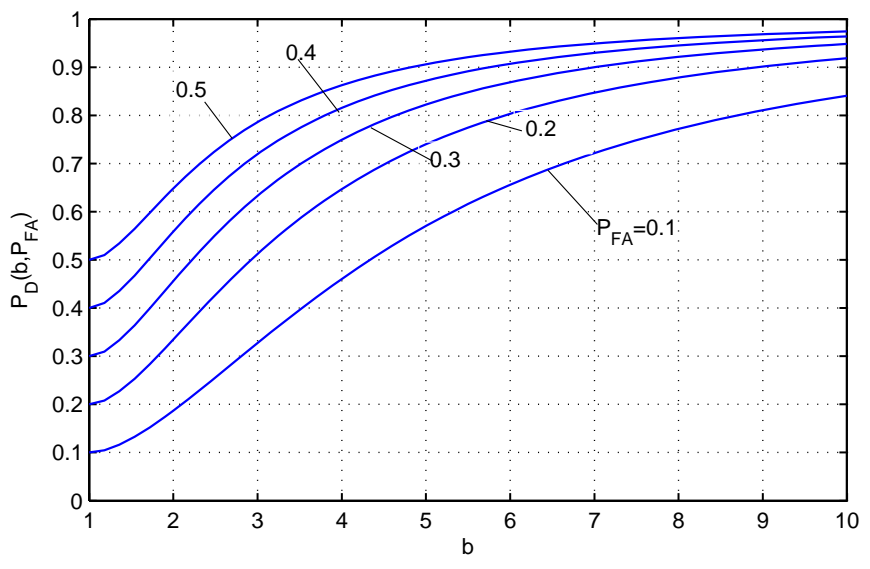

Figure 11. The dependence of the probability of detection on the polarization characteristic of the total signal $b$ with a given $P_{F}$ and $a_{1}=0.3$

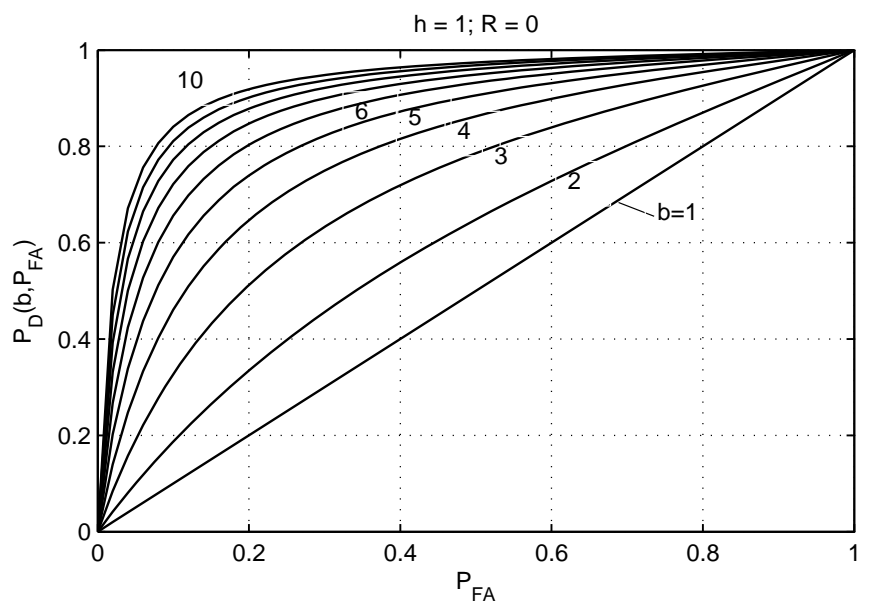

Figure 12. Probability of detection corresponds to given probability of false alarm with $a_{1}=1$ and different values of $b$

Figure 10 shows that if $P_{F}$ increases, the detection range is narrower, and vice versa. The optimum threshold, therefore, depends on $P_{F}$. If $W_{s c+t}(K)$ has the PDF as in Equation (6), $P_{D}$ can be determined from the detection threshold, as described in Equation (16). This equation is received by substituting Equation (15) into Equation (12).

From Equation (16), $P_{D}$ depends on $b$ as illustrated in Figure 11, and on $P_{F}$ as illustrated in Figure 12.

Figure 11 shows that $P_{D}$ depends on the polarization characteristic of the total signal $b$. With the same given $P_{F}$, if $b$ increases, $P_{D}$ also increases. As can be seen in Figure 8, when $b$ increases, the PDF $W_{s c+t}(K)$ is shifted toward -1 . The PDF of $K$ of target + clutter is shifted further away from the PDF of $K$ of the sea clutter. With the same $b$, if $P_{F}$ increase, $P_{D}$ increase as well. This relation is illustrated in Figure 12 and Figure 13.

Figure 13 shows the dependence of $P_{D}$ on $a_{1}$. It can be seen from the figure that $P_{D}$ increases if $a_{1}$ increases. With the same given $P_{F}$, for example $P_{F}=0.2$, if $b$ increases, $P_{D}$ increases. The implication is that the more different the polarimetric parameters of targets to those of the sea clutter, the higher the $P_{D}$ is.

If the polarimetric parameter of target is similar to those of sea clutter, the probability of detection even 


$$
\begin{aligned}
P_{D}= & 1-P_{M}=1-\int_{K_{L}}^{K_{R}} \mathrm{~W}_{s c+t}(K) \mathrm{d} K \\
= & \int_{K_{L}}^{K_{R}} \frac{4 h^{2}(1-K)^{2}}{(1-K)^{2}+h^{2}(1+K)^{2}} \times \exp \left\{-\frac{a_{1}^{2}\left[(1-K)^{2} b^{2}+(1+K)^{2} h^{2}\right]}{2\left[(1-K)^{2}+(1+K)^{2} h^{2}\right]}\right\} \\
& \times\left\{\left[1+\frac{a_{1}^{2}\left[(1-K)^{2}+(1+K)^{2} h^{2} b^{2}\right]}{2\left[(1-K)^{2}+(1+K)^{2} h^{2}\right]}\right] \times I_{0}\left[\frac{a_{1}^{2} b h(1-K)^{2}}{(1-K)^{2}+(1+K)^{2} h^{2}}\right]\right. \\
& \left.+\frac{a_{1}^{2} b h(1-K)^{2}}{(1-K)^{2}+(1+K)^{2} h^{2}} \times I_{1}\left[\frac{a_{1}^{2} b h(1-K)^{2}}{(1-K)^{2}+(1+K)^{2} h^{2}}\right]\right\} \mathrm{d} K
\end{aligned}
$$

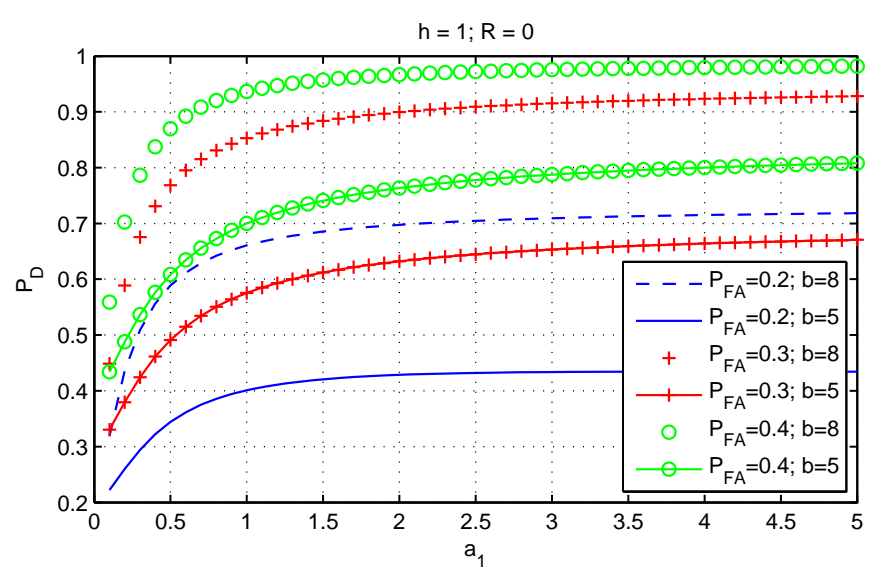

Figure 13. The probability of detection based on $a_{1}$

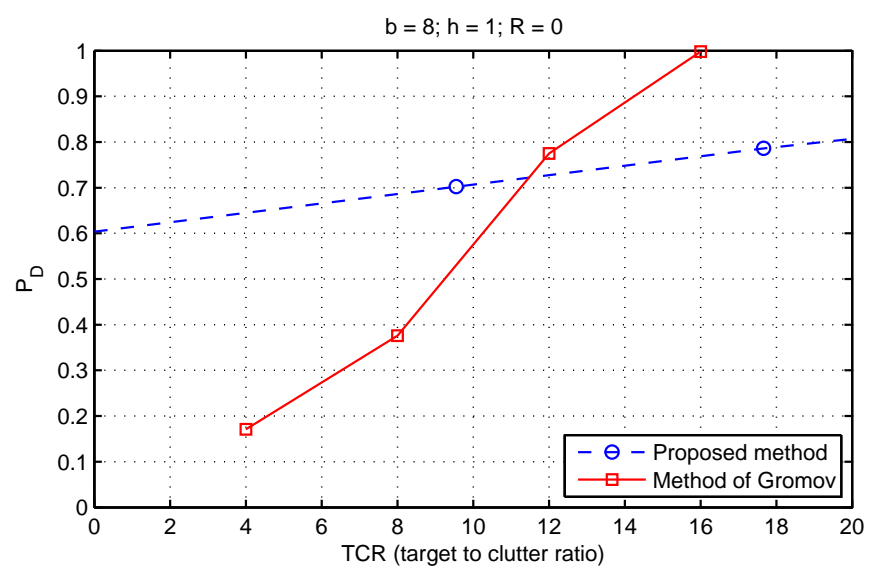

Figure 14. Probability of detection of the Gromov method Vs the proposed method

decreases when $a_{1}$ increases. The reason for that is: if $a_{1}$ increases, the distribution $W(K)$ is narrower, and the distribution of $K$ parameter of both sea clutter and target is similar to that of sea clutter only. This, in turn, results in the increase of miss detection and the decrease of $P_{D}$.

Figure 13 also shows that $P_{D}$ increases significantly when $a_{1}<1$, but it increases negligibly when $a_{1}>1$. This is different from the common methods of target detection based on threshold, in which $P_{D}$ first increases gradually at small $a_{1}$ and then increases significantly at large $a_{1}$ later.
Figure 14 compares $P_{D}$ of the Gromov method $[3,4]$ to the proposed method with the given $P_{F}=0.1$. The total signal is TCR $=a_{1}^{2}+a_{2}^{2}=a_{1}^{2}\left(1+b^{2}\right)$, in which $a_{1}^{2}$ and $a_{2}^{2}=a_{1}^{2} b^{2}$ are TCSs of the 2 orthogonal polarization channels. Table II shows the differences of the proposed method compared with the Gromov' method [4, 13].

It can be seen from the Figure 14 that $P_{D}$ of the proposed method is higher than that of the Gromov when the total signal from target and background clutter is small (e.g. TCR $<11.5 \mathrm{~dB}$ ). If $T C R=4$ $\mathrm{dB}$, the proposed method introduces $P_{D}=0.65$ while the Gromov method introduces $P_{D}=0.18$. In contrast, when TCR increases (e.g. TCR $>11.5 \mathrm{~dB}$ ), the Gromov method shows higher $P_{D}$. This is because the proposed method relies on the polarization feature of the target, which does not strongly depends on the strength of the reflected signal but on the shape, size and the electricphysical nature of the target. The Gromov's method, on the other hand, depends on the polarization and power of the reflected signal. $P_{D}$ of the proposed method depends on $a_{1}, b, h, R$ and $P_{F}$ while that of Gromov method depends on TCR, the given $\varepsilon_{0}$ and $P_{F}$.

\section{Conclusion}

In this paper, the problem of detecting target in background clutter using the eliptic polarimetric parameter is addressed. The PDFs of K parameter for two classes of target: target in sea clutter and only sea clutter have been investigated. Based on those models, a novel method of detecting target in the sea clutter, using interval threshold of polarimetric parameter based on the Neyman-Pearson criteria, is proposed. Simulation results show that $P_{D}$ based on the polarization parameter K not only depends on the strength of returned signal but also strongly depends on the polarization parameters of the target, $b$, and the background clutter, $h$. The comparison of the proposed method and the Gromov method in [4] and [13] is also implemented. The results shows that, at large TCR the Gromov's method introduces higher probability of detection, while at small TCR from $0-11.5 d B$, the proposed method introduces stable $P_{D}$ from $0.6-0.7$, and is higher than that of the Gromov. The proposed method, therefore, could be preferable in situations where the TCR is small. 
Table II

Comparison of the Proposed Method with the Gromov Method

\begin{tabular}{|l|l|}
\hline Gromov method [4] & Proposed approach \\
\hline $\begin{array}{l}\text { - Detect signal with the given elliptic angle of signal of interest } \\
\text { on the internal noise }\end{array}$ & $\begin{array}{l}\text { - Detect target on the background clutter based on the polari- } \\
\text { metric parameters }\end{array}$ \\
\hline $\begin{array}{l}\text { - Polarimetric parameter used: } \\
\varepsilon=0.5 \frac{2 \cdot E_{x} \cdot E_{y} \cdot \sin (\delta)}{E_{x}^{2}+E_{y}^{2}}\end{array}$ & $\begin{array}{l}\text { - Polarimetric parameter used: } \\
K(t)=\frac{\left|p^{R L}(t)\right|-1}{\left|p^{R \dot{L}}(t)\right|+1}\end{array}$ \\
\hline - The interval of detection increases when $P_{F}$ increases & - The interval of detection decreases when $P_{F}$ increases \\
\hline $\begin{array}{l}\text { Detect target when measured polarimetric parameter is in } \\
\text { detection range }\end{array}$ & $\begin{array}{l}\text { - Detect target when measured polarimetric parameter is out of } \\
\text { detection range }\end{array}$ \\
\hline - Can be applied in passive radar systems & $\begin{array}{l}\text { - Can be applied in active radar systems which transmit RHCP } \\
\text { signal and receive RHCP and LHCP orthogonal polarization } \\
\text { channels }\end{array}$ \\
\hline
\end{tabular}

\section{REFERENCES}

[1] G. Valchula and R. Barnes, "Polarization detection of a fluctuating radar target," IEEE Transaction on Aerospace and Electronic System, vol. AES-19, no. 2, pp. 250-256, March 1983.

[2] C. Graves, "Radar polarization power scattering matrix," in Proceedings of the IRE.44, 1956, pp. 248-252.

[3] V. Gromov, G. Sharygin, and M. Mironov, "Method of the radar signals detection and selection using polarization parameter," in Proceeding of the 8th European Radar Conference, Manchester, UK, 2011.

[4] B. Gromov, Patent. 2476903 RF, Method of detection and selection radar signals using polarization sign and device for implementation, Russian Federation, 27.02.2013.

[5] V. Karnjsev, "Polarization contrast of radar target (in russian)," Ph.D. dissertation, Tomck, Russia, 1993.

[6] V. Tatarinov, S. Tatarinov, and L. Ligthart, An Introduction to Radar Signals Polarization Morden Theory. Tomck,Russia: Publ. House of Tomck State University, 2006.

[7] N. Krivin, "Polarization trace and polarization contrast of small scale target (in russian)," Ph.D. dissertation, Tomck, Russia, 2015.

[8] N. Krivin, V. Tatarinov, and S. Tatarinov, "Innovations in radar technologies: Polarization invariants parameter utilization for the problem of radar object detection and mapping," in Proceedings of the First Postgraduate Consortium International Workshop, IICST, Tomck, Russia, October 2011.

[9] L. Ligthart, V. Tatarinov, S. Tatarinov, and E. Pusone, "An effective polarimetric detection of small-scale manmade radar objects on the sea surface," in Proceedings of Microwaves, Radar and Wireless Communications - 14th International Conference on Publication Year, MIKON, vol. 2, Tomck, Russia, 2002.

[10] S. Pozdnyak and V. Melitzky, An Introduction to Statistical Theory of radiowaves polarization (In Russian). Moscow,Russia: Radio Publ. House, 1974.

[11] V. Xlusov, "Measurement of polarimetric parameter using monopulse radar (in russian)," Ph.D. dissertation, Tomck, Russia, 1989.

[12] V. Bogorodsky, D. Kanareikin, and A. Kozlov, The Polarization of Scattered and Thermal Radiation of Earth Surface (In Russian). Lenigrad,Russia: GirometeoIzdat Publ. House, 1974.

[13] V. Gromov, "Detection and selection of ground base $x-$ band radar signals vector in the pasive monitoring space system (in russian)," Ph.D. dissertation, Tomck, Russia, 2014.

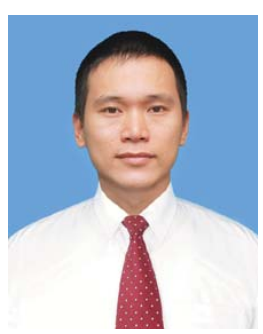

Trong Hung Pham received the B.S from Moscow Aviation Institute, Russia in 2007 and M.S. degrees in electronic engineering from Le Qui Don Technical University, Hanoi, Vietnam, in 2010. He is currently a Lecturer and PhD student with the Department of Electronic Warfare, Faculty of Radio Electronics, Le Qui Don Technical University. His research interests include polarimetric radar and signal processing.

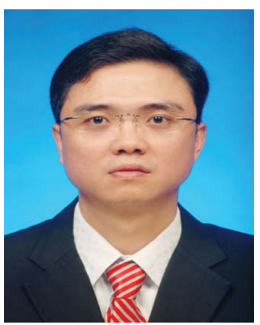

Minh Nghia Pham received the B.S. and M.S. degrees in electronic engineering from Le Qui Don Technical University, Hanoi, Vietnam, in 2005 and 2008, respectively, and the Ph.D. degree in information and communication engineering from the Harbin Institute of Technology (HIT), Harbin, China, in 2014. He is currently a Lecturer with the Department of Circuits and Signal Processing, Faculty of Radio Electronics, Le Qui Don Technical University. His research interests include synthetic aperture radar (SAR) image processing, polarimetric SAR, polarimetric SAR interferometry, and signal processing.

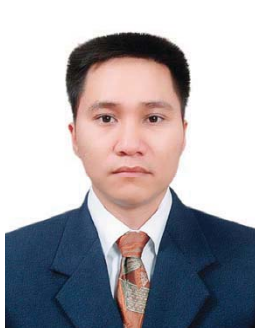

Trung Thanh Nguyen has been a lecturer in Radio-Electronics Engineering Faculty, Le Qui Don University since 2002. He received the M.Eng (Electronics) in 2009, and Ph.D degree in electronics engineering in 2015 at LaTrobe University, Australia. His research interests include radar system, signal processing and FPGA implementation. 